\title{
Effects of Dietary Composition and Exercise Timing on Energy Expenditure and Substrate Utilization in Healthy Young Women
}

\author{
Tatsuhiro MATSUo, ${ }^{1, *}$ Hiroshi Sumida, ${ }^{1}$ Hiroko Jimbo, ${ }^{1}$ Yukio SHIRAISHI, ${ }^{2}$ \\ Tatsuhito FUKUOKA, ${ }^{2}$ and Masashige SUZUKI ${ }^{3}$ \\ ${ }^{1}$ Division of Nutrition and Biochemistry, Sanyo Women's College, Hatsukaichi 738, Japan \\ ${ }^{2}$ Section of Clinical Research and Laboratory, JA Hiroshima General Hospital, \\ Hatsukaichi 738, Japan \\ ${ }^{3}$ Institute of Health and Sport Sciences, University of Tsukuba, Tsukuba 305, Japan
}

(Received December 14, 1995)

\begin{abstract}
Summary Effects of dietary composition (high fat, FAT; or high carbohydrate, $\mathrm{CHO}$ ) and exercise timing (preprandial exercise, Ex-; or postprandial exercise, -Ex) on postprandial energy expenditure and substrate utilization were studied in seven women aged 19-20 years. The experimental protocol included four different sessions (Ex-FAT, ExCHO, FAT-Ex, and CHO-Ex). The FAT and CHO diets provided 48 and $5 \%$ as fat, respectively. On the experimental days, subjects were fed a meal containing the same caloric energy at lunch time and they exercised for 30 min on a bicycle ergometer at an intensity of $60 \% \mathrm{VO}_{2 \max }$ at pre- or post-meal followed by rest for $3 \mathrm{~h}$. The values of oxygen consumption were $1,559,1,628,1,526$, and $1,557 \mathrm{ml} \cdot \mathrm{kg}^{-1} \cdot 4 \mathrm{~h}^{-1}$ in Ex-FAT, Ex-CHO, FAT-Ex, and CHO-Ex groups, respectively. Total levels of serum insulin were $7.6,13.7,4.5$, and $7.8 \mu \mathrm{U} \cdot \mathrm{ml}^{-1} \cdot \mathrm{min} \cdot 10^{-3}$, respectively. These results suggest that preprandial exercise, especially before $\mathrm{CHO}$ diet intake, has an advantage for increasing oxygen consumption. This advantage might be related to rates of substrate ("futile") cycling and insulinemic responses at rest and during exercise.
\end{abstract}

Key Words: high fat diet, high carbohydrate diet, exercise timing, oxygen consumption, respiratory exchange ratio

Association with exercise training has useful effects on diet therapy for obese individuals owing to the increases in energy expenditure and substrate metabolism

* To whom correspondence should be addressed. 
$[1,2]$. Many persons who maintain a normal body weight also have a habit of daily exercise for building up their health. In Japan, some workers and students take light exercise at a noon recess, either before or after lunch. From a practical viewpoint, however, it is unclear which exercise, before or after a meal, is better to maximize its thermogenic effect. It has been reported that exercise before a meal enhances the thermogenic response to meals in humans [3-6], but the effect of exercise after a meal on diet-induced thermogenesis is unclear. Segal and Gutin [7] and Segal et al. [8-10] have reported that enhanced postprandial energy expenditure was found during and after exercise. However, other researchers have believed that there is no relationship between exercise and diet-induced thermogenesis [11$14]$.

In addition, the amount of fat in the diet is suggested to be an important factor affecting body fat accumulation [15]. It is well known that diet-induced thermogenesis is larger with the intake of a high carbohydrate diet than with that of a high fat diet during the resulting condition [16]. However, it is unclear whether the difference of thermic effect between a high carbohydrate diet or a high fat diet was enhanced by the exercise. There are few studies that have examined the effects of the types of diet, high fat or high carbohydrate, on postprandial energy expenditure during exercise in humans [17] and in rats [18, 19]. We previously reported that postprandial energy expenditure during exercise was higher in rats fed a high carbohydrate diet than in those fed a high fat one, which could have been related to the increase in glycogen storage in the former [19]. In the previous study, we did not examine the thermogenic effects of meal and exercise during rest after prolonged exercise.

On the other hand, it is well known that moderate exercise reduces the postprandial glycemic and insulinemic responses in normal and NIDDM subjects [20], and decreases serum triacylglycerol levels [21]. Hyperinsulinemia accelerates body fat accumulation [22] due to an increase in lipogenesis in the liver and adipose tissues, and lipid uptake in adipose tissues from the bloodstream. While exercise plays an important role in preventing the excess accumulation of body fat, there are few studies that have examined the effects of interactions between dietary composition and exercise timing on the glycemic or insulinemic response.

The purpose of this study was to examine the effect of dietary composition and exercise timing on subsequent energy expenditure and substrate utilization with respect to hormonal responses in young healthy Japanese women.

\section{MATERIALS AND METHODS}

Subjects. Seven young Japanese women (aged 19-20 years) who did not have the habit of daily exercise were recruited from Sanyo Women's College (Hiroshima, Japan) to participate in this study. All procedures were approved in advance by the Human Use Committee of Sanyo Women's College and were followed in accordance with the Helsinki Declaration of 1975, as revised in 1983. 
After a detailed explanation of this study, each subject gave her informed written consent. Subjects were diagnosed as being free of disease by a medical examination at Hiroshima General Hospital before the study. Physical characteristics of the subjects are shown in Table 1 . The percentage of body fat was measured with a bioelectrical impedance analyzer (Model TBF-102, Tanita Ltd., Tokyo). Maximal oxygen consumption $\left(\dot{\mathrm{VO}}_{2 \max }\right)$ was determined by the method of Astrand and Rodahl [23] by use of a bicycle ergometer (Monark, Varberg, Sweden). The $\dot{\mathrm{VO}}_{2 \max }$ values of subjects were average or high compared with the standard value of 20-year-old females.

Experimental design. During the period of the study, each subject maintained a normal life style and ate ad libitum except for the day before the experimental period, in which each subject ate the same supper $\left(50 \mathrm{~kJ} \cdot \mathrm{kg}^{-1}\right.$ body weight $)$ at 19:00 h. Subjects fasted overnight, and they entered the experimental room at 9:00 $\mathrm{h}$ where they rested until the start of experiments at 11:30 h. The experimental protocol, shown in Fig. 1, included four different sessions. All experiments were performed in the preovulatory phase on the 8th-12th day after the onset of menstration [24]. The experimental sessions were divided into two types: preprandial exercise (Ex-) and postprandial exercise (-Ex). They were then divided into two subtypes: high fat diet (FAT) and high carbohydrate diet (CHO). The four types of experimental sessions were labeled Ex-FAT, Ex-CHO, FAT-Ex, and CHO-Ex (Fig. 1). The FAT diet provided 37, 48, and 15\% of energy as carbohydrate, fat and protein, respectively, whereas the $\mathrm{CHO}$ diet provided 80,5 , and $15 \%$, respectively. The energy of the experimental diets were 8,440 and $6,350 \mathrm{~J} \cdot \mathrm{g}^{-1}$ as FAT and $\mathrm{CHO}$ diets, respectively. The experimental diet consisted of rice (Japonica), milk, low fat milk, yoghurt, cheese, and butter. The fatty acid composition of the two experimental diets was nearly the same.

On the experimental days, subjects were fed a meal containing the same amount of energy (57 $\mathrm{kJ} \cdot \mathrm{kg}^{-1}$ body weight) at 12:45-13:00 h (Ex-FAT, Ex-CHO) or 12:00-12:15 h (FAT-Ex, CHO-Ex). Subjects exercised for $30 \mathrm{~min}$ on a bicycle ergometer (Monark, Varberg, Sweden) at an intensity of $60 \% \dot{\mathrm{VO}}_{2 \max }$ at $12: 00$ 12:30 h (Ex-FAT, Ex-CHO) or 12:30-13:00 h (FAT-Ex, CHO-Ex). The subjects

Table 1. Characteristics of subjects.

\begin{tabular}{cccccc}
\hline Subject & $\begin{array}{c}\text { Age } \\
(\text { years })\end{array}$ & $\begin{array}{c}\text { Height } \\
(\mathrm{cm})\end{array}$ & $\begin{array}{c}\text { Weight } \\
(\mathrm{kg})\end{array}$ & $\begin{array}{c}\text { Body fat } \\
(\%)\end{array}$ & $\begin{array}{c}\dot{\mathrm{VO}}_{2 \max } \\
(\mathrm{ml} /(\mathrm{kg} \cdot \min ))\end{array}$ \\
\hline 1 & 19 & 157.0 & 47.1 & 21.3 & 43.8 \\
2 & 20 & 173.5 & 65.0 & 26.0 & 38.1 \\
3 & 19 & 163.0 & 60.4 & 28.4 & 44.2 \\
4 & 20 & 159.0 & 64.2 & 36.7 & 33.4 \\
5 & 19 & 158.0 & 51.1 & 24.5 & 47.5 \\
6 & 20 & 150.0 & 41.5 & 21.0 & 49.5 \\
7 & 19 & 155.5 & 46.6 & 25.5 & 40.7 \\
Mean \pm SD & $19.6 \pm 0.5$ & $159.4 \pm 7.3$ & $53.7 \pm 9.4$ & $26.2 \pm 5.3$ & $42.5 \pm 5.5$ \\
\hline
\end{tabular}

Vol. 20, No. 2, 1996 


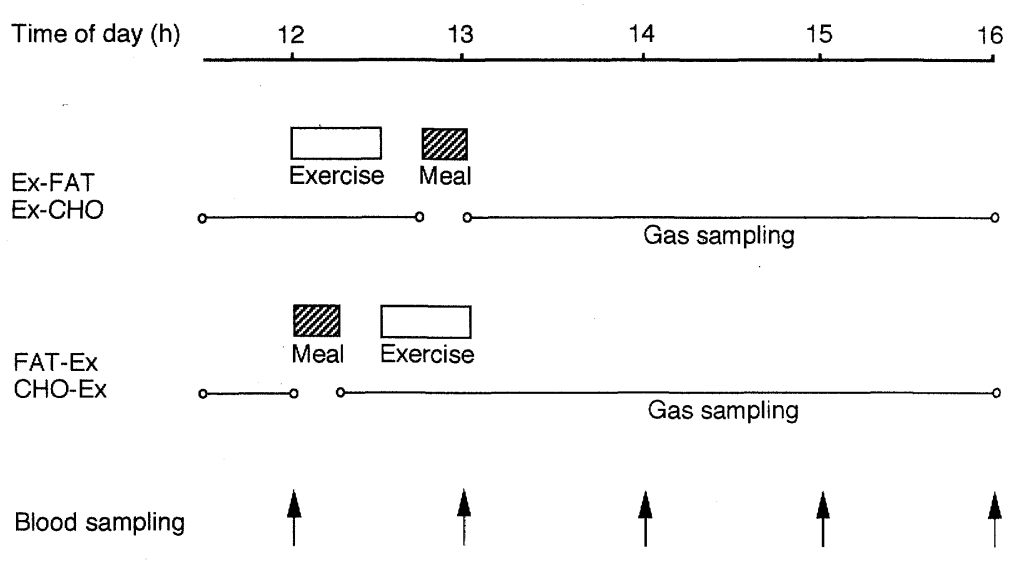

Fig. 1. The experimental design. For definitions, see text.

then rested for $3 \mathrm{~h}$. During rest and exercise, oxygen uptake and respiratory exchange ratio $(R)$ were measured $(11: 30-16: 00 \mathrm{~h})$. Blood samples were collected from the cephalic vein at the level of the forearm to obtain serum and plasma at $12: 00,13: 00,14: 00,15: 00$, and 16:00 $\mathrm{h}$. All procedures were performed in the experimental room under the same conditions (temperature: $22 \pm 1^{\circ} \mathrm{C}$, humidity: $60 \%)$.

Measurements. Indirect calorimetry was performed by the ventilated hood technique. For measurement of oxygen uptake and $R$, the subjects wore face masks (Takei Ltd., Tokyo) continuously throughout $270 \mathrm{~mm}$ of each experiment, except for meal time $(15 \mathrm{~min})$. All the expired gas was collected into a Douglas bag (Takei Ltd., Tokyo), and the bag was changed every 15 min during rest and every 5 min during exercise. The concentrations of oxygen and carbon dioxide in the expired collected gas were immediately analyzed by a gas analyzer (Model RAS30, RAS-31, AIC Co., Tokyo).

Plasma glucose was assayed by a glucose analyzer with electrochemical detection (Model GA-1140, Kyoto Daiichi Co., Kyoto) and a kit (GLC-C) purchased from Kokusai Shiyaku Co., Kobe. Serum triacylglycerol (TG) and free fatty acids (FFA) were determined enzymatically with kits (TG-A, NEFA-V5) purchased from Kokusai Shiyaku Co., Kobe and Nippon Shoji Co., Osaka, respectively. Serum immunoreactive insulin (IRI) was determined by radio-immunoassay (two-antibody system) with kits (Insulin 'Eiken') purchased from Eiken Chemical Co., Tokyo.

Statistical analysis. Statistical analysis was conducted using a personal computer (Macintosh LC 575, Apple Japan, Inc.) with a statistical package program (Stat View 4.02, Abacus Concepts, Inc., Berkeley, CA). The effects of dietary composition and exercise timing were analyzed by repeated measures of the analysis of variance (ANOVA) followed by paired comparisons using $t$-tests. 


\section{RESULTS}

\section{Oxygen consumption and $R$ during rest and exercise}

Oxygen consumption during rest and exercise was measured for $4 \mathrm{~h}$ to assess the thermogenic effects of the diets and exercise (Fig. 2). The average values of oxygen consumption are shown in Fig. 3. Oxygen consumption for 12:00-13:00 h (meal and exercise time) did not differ among the four sessions. The total values of oxygen consumption for 12:00-16:00 h were higher in the case of $\mathrm{CHO}$ diet intake or preprandial exercise (Figs. 2 and 3). For the CHO diet, oxygen consumption was significantly higher during the preprandial exercise than during the postprandial one $(p<0.05)$. Regardless of exercise timing, oxygen consumption

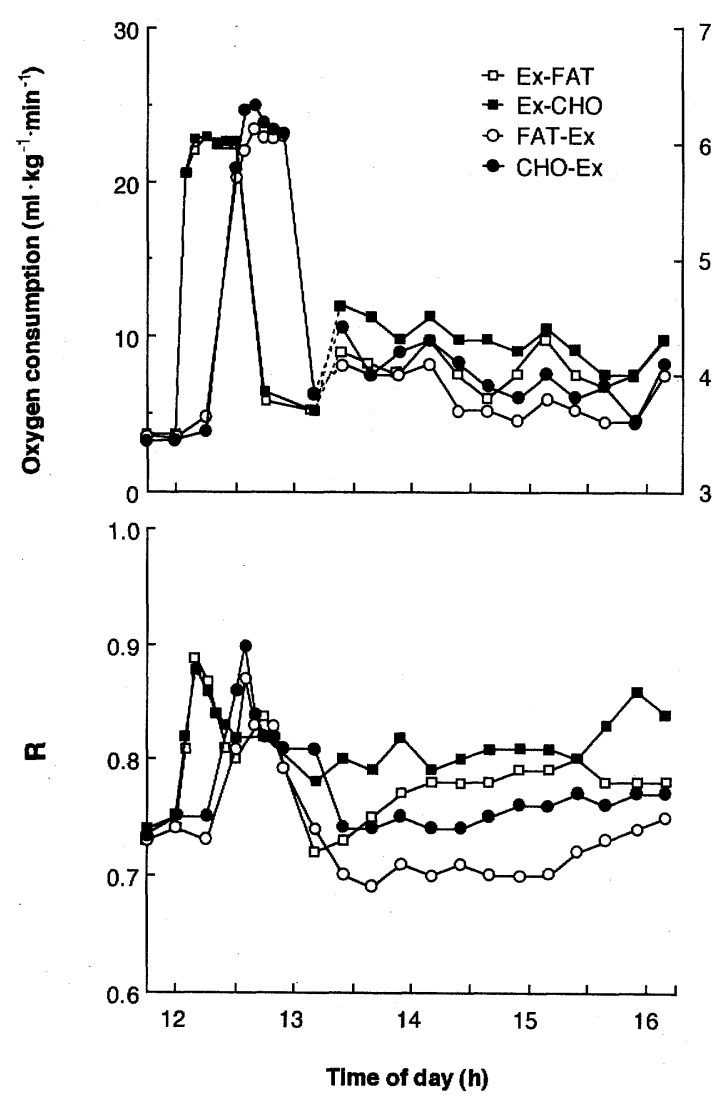

Fig. 2. Oxygen consumption (top) and respiratory exchange ratio ( $R$, bottom) at rest and exercise for Ex-FAT, Ex-CHO, FAT-Ex, and CHO-Ex groups (for definitions see text). In the top figure, the left vertical axis is for 12:00-13:00 h; had the right axis, for 13:0016:00 h. Oxygen consumption and carbon dioxide production were measured in the sessions, Ex-FAT, Ex-CHO, FAT-Ex, and CHO-Ex. The $R$ was calculated from these values. Each point represents the mean value for 7 subjects.

Vol. 20, No. 2, 1996 
was significantly higher in the case of the CHO diet intake than in the FAT diet intake $(p<0.05)$. On the other hand, the $R$ values for 13:00-16:00 h appeared to be higher with the CHO diet or preprandial exercise (Fig. 2). The average values for 12:00-13:00 $\mathrm{h}$ did not differ among the four sessions (mean values; $0.82 \pm 0.01$ ). The average values of $R$ for the experimental period were $0.80,0.82,0.76$, and 0.79 for Ex-FAT, Ex-CHO, FAT-Ex, and CHO-Ex groups, respectively. Irrespective of exercise timing, he mean values of $R$ was significantly higher in the CHO diet ( $p<$ $0.05)$. On the other hand, postprandial exercising decreased $R$ for both diets.
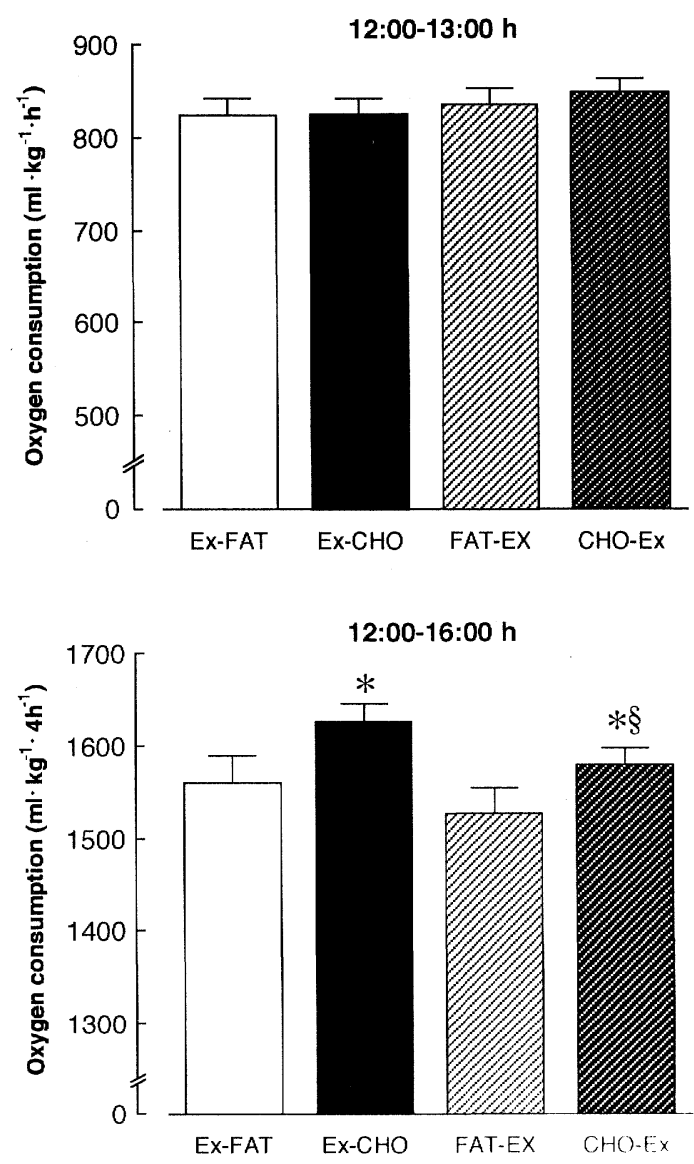

Fig. 3. The sum of oxygen consumption at 12:00-13:00 h (top) and at 12:00-16:00 h (bottom) in Ex-FAT, Ex-CHO, FAT-Ex, and CHO-Ex groups. Each value (mean and SEM) was calculated from the data in Fig. 2. *Statistically significant difference from the FAT diet. ${ }^{8}$ Statistically significant difference from the value of preprandial exercise. Differences with $p<0.05$ (repeated measures of ANOVA followed by paired comparisons with the $t$-test). 
Concentrations of plasma glucose, serum IRI, TG, and FFA

Plasma glucose and serum IRI concentrations increased after meal ingestion and exercise (Fig. 4). Postprandial exercise compared with preprandial exercise induced a reduction in total area of glucose which became statistically significant $(p<0.05)$ after the CHO diet intake, as shown in Fig. 4. The total area of IRI was significantly higher for the CHO diet intake than for the FAT diet intake $(p<$ 0.05 ). In the case of CHO diet intake, the total area of IRI was significantly lower with postprandial exercise $(p<0.05)$. Exercise after meals decreased concentrations of plasma glucose and serum IRI compared with exercise before meals. Figure 5 shows that the serum TG concentration increased after the FAT diet intake, although the $\mathrm{CHO}$ diet intake did not affect the TG concentration. The total area of TG was significantly higher for the FAT diet intake than for the CHO diet one in the case of postprandial exercising $(p<0.05)$. The serum FFA concentration
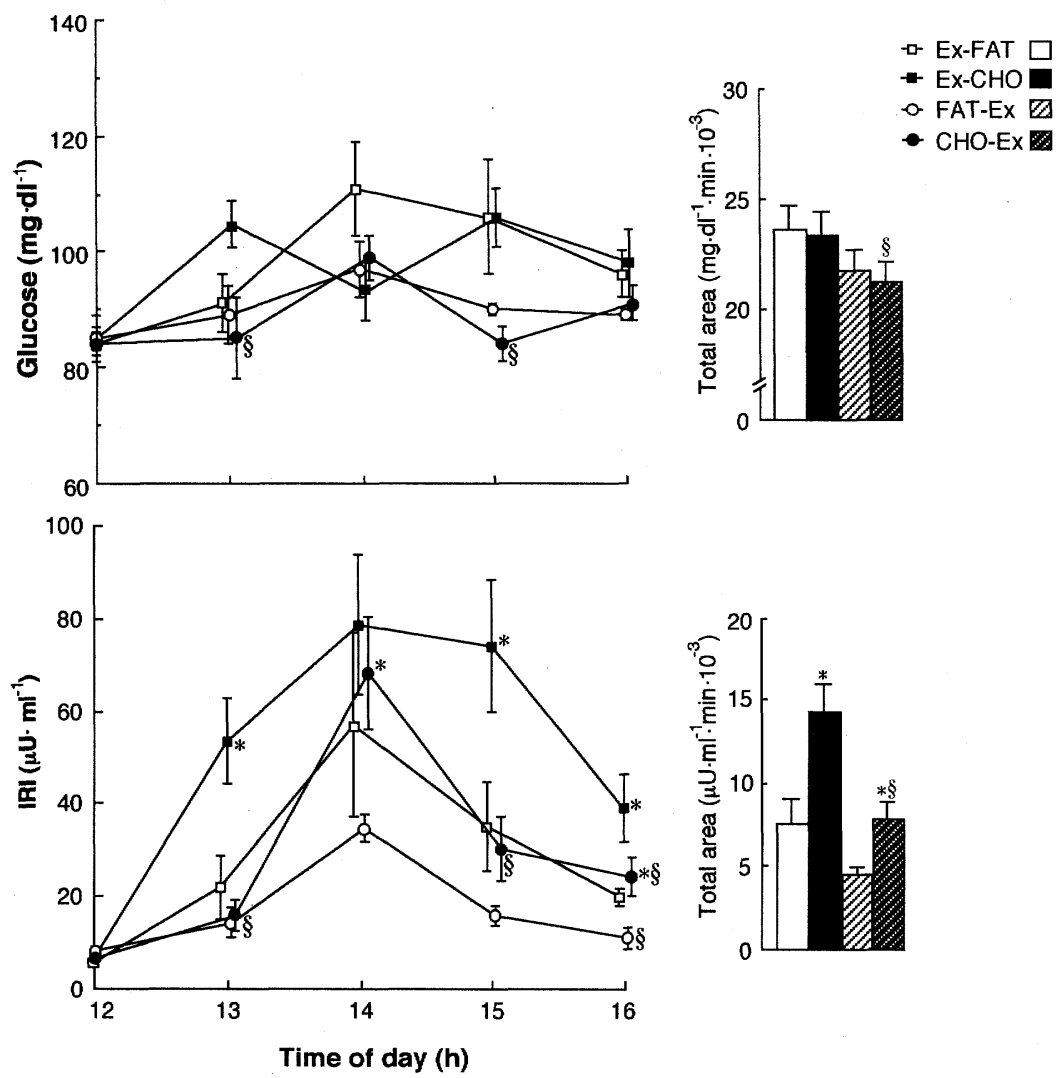

Fig. 4. Serum glucose and IRI concentrations in Ex-FAT, Ex-CHO, FAT-Ex, and CHOEx groups. Total area was calculated from the left figure. Values are means and SEM for 7 subjects. ${ }^{*}$ Statistically significant difference from the FAT diet. ${ }^{\$}$ Statistically significant difference from the value of preprandial exercise. Differences with $p<0.05$ (repeated measures of ANOVA followed by paired comparisons with the $t$-test).

Vol. 20, No. 2, 1996 

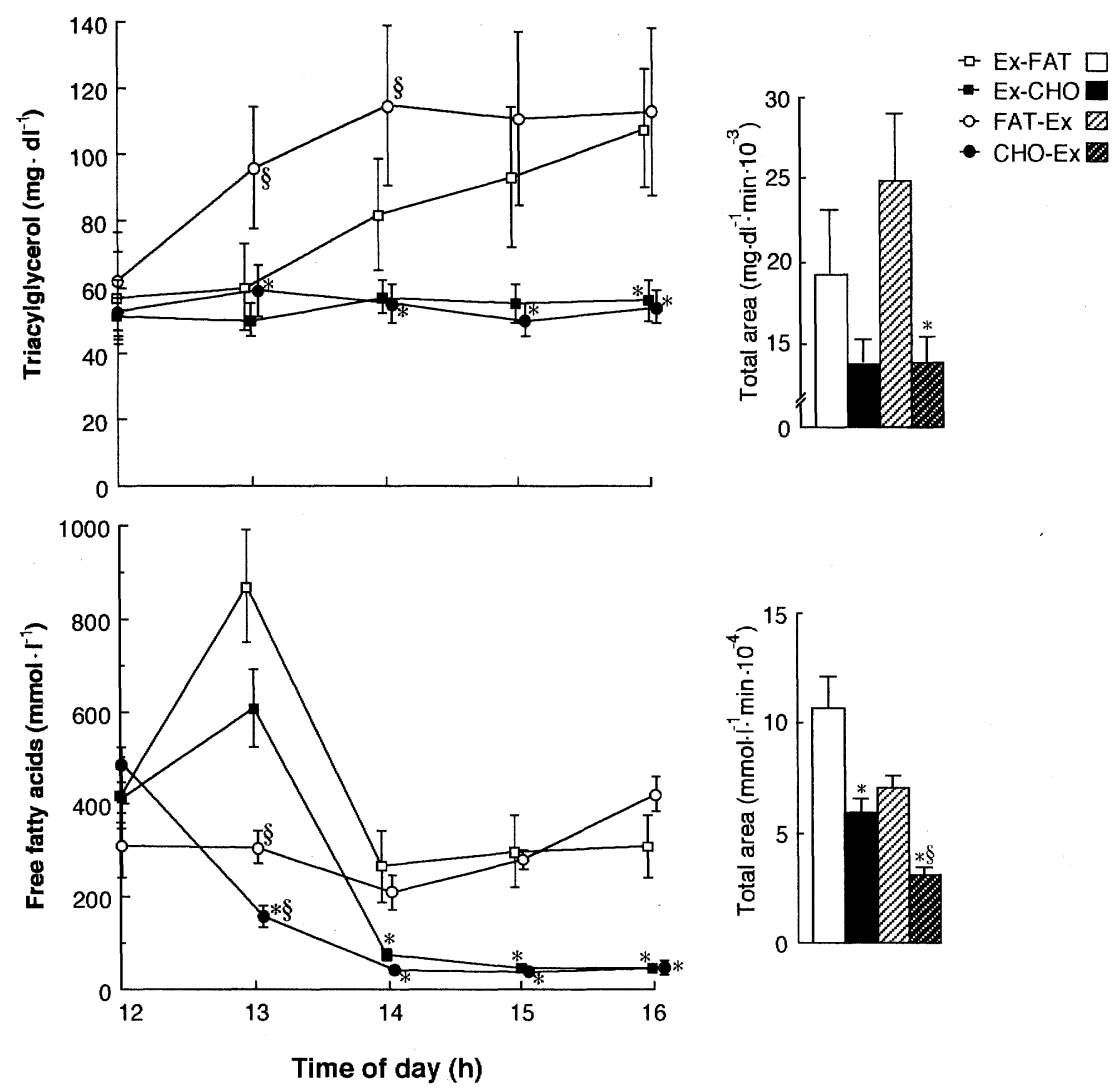

Fig. 5. Serum TG and FFA concentrations in Ex-FAT, Ex-CHO, FAT-Ex, and CHO-Ex groups. Total area was calculated from the left figure. Values are means and SEM for 7 subjects. *Statistically significant difference from the FAT diet. ${ }^{\S}$ Statistically significant difference from the value of preprandial exercise. Differences with $p<0.05$ (repeated measures of ANOVA followed by paired comparisons with the $t$-test).

was decreased by intake of CHO diet at all times (Fig. 5). The total area of serum FFA was significantly lower for the $\mathrm{CHO}$ diet intake regardless of exercise timing $(p<0.05)$. In preprandial exercise sessions, serum FFA concentrations increased temporarily at 13:00 $\mathrm{h}$.

\section{DISCUSSION}

In the present study, oxygen consumption at rest and during exercise for $4 \mathrm{~h}$, especially for $3 \mathrm{~h}$ (after lunch time), was higher in the case of the CHO diet intake or preprandial exercise than in that of the FAT diet intake or postprandial exercise. The oxygen consumption for the experimental period is the result of at least four components: the basal metabolic rate, increase during exercise, diet- 
induced thermogenesis, and excess post-exercise oxygen consumption. We did not examine the occasion of no meal or no exercise in this experiment, as our present study was considered on the premise of exercise and meal intake taking place within $1 \mathrm{~h}$ of each other.

The mean values of oxygen consumption during exercise were $662,662,668$, and $706 \mathrm{ml} \cdot \mathrm{kg}^{-1} \cdot 30 \mathrm{~min}^{-1}$ for Ex-FAT, Ex-CHO, FAT-Ex, and CHO-Ex, respectively. We had previously reported that postprandial oxygen consumption during exercise $(3 \mathrm{~h})$ was higher in rats fed a high carbohydrate diet than in those fed a high fat diet [19], and the findings of the present study not only correspond with these results in rats but also show consistency between humans and rats concerning energy expenditure during exercise. The difference in oxygen consumption during exercise among the four cases was slight and oxygen consumption from 12:00-13:00 $\mathrm{h}$ was not significantly different. The present findings on healthy humans suggest that the contribution of diet and exercise on energy expenditure is more important in the resting time after a meal and exercise than during exercise, as exercise cannot be performed over a long period of time for relatively sedentary humans.

Total carbohydrate utilization can be estimated from oxygen consumption and $R$ for $4 \mathrm{~h}$ by use of the equation

$$
G=\dot{\mathrm{V}_{2}}(R-0.7) \times 1.22 / 0.30 \times 0.91 \quad[25],
$$

where $G$ is the carbohydrates of glycosyl units used at rest and during exercise (Table 2). The results showed that carbohydrate oxidation appeared to be higher in the case of the CHO diet intake and preprandial exercise than in that of the FAT diet intake and postprandial exercise. Since increased carbohydrate oxidation causes enhanced liver and muscle glycogen synthesis [19], the present findings suggest that rates of substrate ("futile") cycling were increased by the CHO diet intake and preprandial exercise and consequently extra energy was required [26, 27].

The CHO diet intake increased the serum insulin level compared with the FAT diet intake, especially when exercise was performed before a meal. Insulin increases the thermogenic effects of increased sympathetic activity in the brown adipose tissue and skeletal muscles [28]. In the present study, since the results of the total area of serum insulin were parallel with those of total oxygen consumption, the enhancement of energy expenditure in the case of the $\mathrm{CHO}$ diet or preprandial exercise may be also caused by the increase in serum insulin level. On the other hand, insulin increases lipid uptake in adipose tissues from the blood, and enhances body fat deposits. However, serum TG levels were lower in Ex-CHO and CHO-Ex in this experiment, probably because lipogenesis and TG release in the liver are lower in humans compared with their values for animals [29]. These results suggest that a high carbohydrate diet intake would not cause as much body-fat accumulation as a high fat diet intake, especially if accompanied with exercise.

The concentrations of serum TG and FFA were higher in the case of the FAT diet intake than in that of the CHO diet intake. In the sessions of the FAT diet, 
Table 2. Estimation of carbohydrate utilization from oxygen consumption and respiratory exchange ratio $(R)$.

\begin{tabular}{cccccc}
\hline & $\begin{array}{c}\text { Time of day } \\
(\mathrm{h})\end{array}$ & $\begin{array}{c}\dot{\mathrm{VO}}_{2} \\
(\mathrm{ml} /(\mathrm{min} \cdot \mathrm{kg}))\end{array}$ & $R$ & $\begin{array}{c}\text { Carbohydrate } \\
(\mathrm{mg})\end{array}$ & \\
\hline Ex-FAT & $12-13$ & 13.8 & 0.82 & 23.8 & 36.2 \\
& $13-16$ & 4.1 & 0.78 & 12.4 & \\
Ex-CHO & $12-13$ & 13.8 & 0.83 & 25.8 & 48.1 \\
& $13-16$ & 4.3 & 0.82 & 22.3 & \\
FAT-Ex & $12-13$ & 14.0 & 0.80 & 20.2 & 21.8 \\
& $13-16$ & 3.8 & 0.71 & 1.6 & \\
CHO-Ex & $12-13$ & 14.3 & 0.83 & 26.8 & 37.2 \\
& $13-16$ & 4.0 & 0.76 & 10.4 & \\
\hline
\end{tabular}

$\dot{\mathrm{V}} \mathrm{O}_{2}$, Oxygen consumption. The body weight of the subjects averaged $53.7 \mathrm{~g}$. Carbohydrate ( $G$ ) was calculated from the equation $G=\mathrm{V}_{2}(R-0.7) \times 1.22 / 0.30 \times 0.91$ [25], where $G$ is carbohydrates of glucosyl units, $\dot{\mathrm{V}} \mathrm{O}_{2}$ is $\dot{\mathrm{V}}_{2}$ in steady-state (liter/min, multiplied by the experimental duration in $\mathrm{min}), R$ is respiratory exchange ratio in steady-state $(1.22 \mathrm{~kJ} /$ liter of $\mathrm{O}_{2}$ burnt per glucosyl unit), and 0.91 is $\mathrm{kJ} / \mathrm{g}$ of glucosyl units burnt with $\mathrm{O}_{2}$.

the total area of serum TG was lower and FFA was higher for Ex-FAT than for FAT-Ex, although the values were not significant. These results suggest that lipolysis and lipid uptake in adipose tissues, the turnover of stored fat, are higher for Ex-FAT than for FAT-Ex, and this may be one of the reasons that oxygen consumption was higher in the former. In this context, it should be noted that serum insulin levels were higher in the Ex-FAT session.

Finally, the estimated values of diet- and exercise-induced energy expenditure [30] during the experimental periods were 14.6, 15.4, 13.6, and $14.8 \mathrm{~kJ} \cdot \mathrm{kg}^{-1}$ for ExFAT, Ex-CHO, FAT-Ex, and CHO-Ex, respectively. These results showed that light exercise before or after a meal resulted in considerable caloric energy loss. From these points of view, pre- or postprandial exercise at a noon recess on weekdays should be available for body conditioning and shaping up, and further effects may be expected if contents of diet and exercise timing are taken into consideration as much as possible.

In summary, preprandial exercise, compared with postprandial exercise, showed higher subsequent oxygen consumption, when a high carbohydrate diet was consumed; and this might be related to rates of substrate cycling and insulinemic responses at rest and during exercise.

We thank Dr. Seiryo Takashina, former Medical Director of JA Hiroshima General Hospital, for his invaluable assistance with various aspects of the laboratory studies. We also thank Ms. Akemi Shinya, Ms. Miyuki Satoh, and Ms. Mikiko Shinya for their skillful assistance. We would also like to convey our appreciation to the subjects who participated faithfully in the experiment. 


\section{REFERENCES}

1. LeBlanc, J.(1988): Exercise training and energy expenditure, in Diet and Obesity, ed. by Bray, G., LeBlanc, J., Inoue, S., and Suzuki, M., Karger, Basel, pp. 181-190.

2. Sweeney, M.E., Hill, J.O., Heller, P.A., Baney, R., and DiGirolamo, M. (1993): Severe vs moderate energy restriction with and without exercise in the treatment of obesity: Efficiency of weight loss. Am. J. Clin. Nutr., 57, 127-134.

3. Bielinski, R., Schutz, Y., and Jequier, E. (1985): Energy metabolism during the postexercise recovery in man. Am. J. Clin. Nutr., 42, 69-82.

4. Maehlum, S., Grandmantagne, M., Newsholme, E.A., and Sejersted, O.M. (1986): Magnitude and duration of excess postexercise oxygen consumption in healthy young subjects. Metabolism, 35, 425-429.

5. Young, J.C., Treadway, J.L., Balon, T.W., Gavras, H.P., and Ruderman, N.B. (1986): Prior exercise potentiates the thermic effect of a carbohydrate load. Metabolism, 35, 1048-1053.

6. Poehlman, E.T., and Horton, E.S. (1989): The impact of food intake and exercise on energy expenditure. Nutr. Rev., 47, 129-137.

7. Segal, K.R., and Gutin, B. (1983): Thermic effects of food and exercise in lean and obese women. Metabolism, 32, 581-589.

8. Segal, K.R., Presta, E., and Gutin, B. (1984): Thermic effects of food during graded exercise in normal weight and obese men. Am. J. Clin. Nutr., 40, 995-1000.

9. Segal, K.R., Gutin, B., Nyman, A.M., and Pi-Sunyer, F.X. (1985): Thermic effects of food at rest, during exercise, and after exercise in lean and obese men of similar body weight. $J$. Clin. Invest., 76, 1107-1112.

10. Segal, K.R., Chun, A., Coronel, P., and Valdez, V. (1992): Effects of exercise mode and intensity on postprandial thermogenesis in lean and obese men. J. Appl. Physiol., 72, 17541763.

11. Dallosso, H.M., and James, W.P.T. (1984): Whole-body calorimetry studies in adult men. Br. J. Nutr., 52, 65-72.

12. Hickson, J.F., Hartung, G.F., Jr., Pate, T.D., Kendall, S.C., McMahon, J.C., and Moore, C.M. (1986): Effect of short-term energy intake level and exercise on oxygen consumption in men. Eur. J. Appl. Physiol., 55, 198-201.

13. Pacy, P.J., Barton, N., Webster, J.D., and Garrow, J.S. (1985): The energy cost of aerobic exercise in fed and fasted normal subjects. Am. J. Clin. Nutr., 42, 764-768.

14. Schutz, Y., Bessard, T., and Jequier, E. (1987): Exercise and postprandial thermogenesis in obese women before and after weight loss. Am. Clin. J. Nutr., 45, 1424-1432.

15. Flatt, J.P. (1976): The difference in storage capacities for carbohydrate and for fat, and its implications in the regulation of body weight. Ann. N.Y. Acad. Sci., 449, 104-123.

16. Jequier, E. (1986): Influence of nutrient administration on energy expenditure in man. Clin. Nutr., 5, 181-186.

17. Abbott, W.G.H., Howard, B.V., Ruotolo, G., and Ravussin, E. (1990): Energy expenditure in humans effects of dietary fat and carbohydrate. Am. J. Physiol., 258, E347-E351.

18. Gleeson, M., and Waring, J.J. (1986): Influence of diet on the storage, mobilization and utilization of energy reserves in trained and untrained rats. Comp. Biochem. Physiol., 85, 411-415.

19. Saitoh, S., Matsuo, T., and Suzuki, M. (1993): The effects of a high carbohydrate diet on postprandial energy expenditure during exercise in rats. Eur. J. Appl. Physiol., 66, 445-450.

20. Tremblay, A., Nadeau, A., Despres, J.P., St.-Jean, L., Theriault, G., and Bouchard, C. (1990): Long-term exercise training with constant energy intake. 2: Effects on glucose metabolism and resting energy expenditure. Int. J. Obes., 14, 75-84.

21. Pavlou, K.N., Whatley, J.E., Jannace, P.W., DiBartolomeo, J.J., Burrows, B.A., Duthie, E.A.M., and Leman, R.H. (1989): Physical activity as a supplement to a weight-loss dietary regimen. Am. J. Clin. Nutr., 49, 1110-1114. 
22. Shimomura, Y., Tamura, T., and Suzuki, M. (1990): Less body fat accumulation in rats fed a safflower oil diet than in rats fed a beef tallow diet. J. Nutr., 120, 1291-1296.

23. Astrand, P.O., and Rodahl, K. (1986): Textbook of Work Physiology, McGraw Hill, New York.

24. Ferraro, R., Lillioja, S., Fontvieille, A.M., Rising, R., Bogardus, C., and Ravussin, E. (1992): Lower sedentary metabolic rate in women compared with men. J. Clin. Invest., 90, $780-784$.

25. Gorostiaga, E.M., Czerwinski, S.M., and Hickson, R.C. (1988): Acute glucocorticoid effects on glycogen utilization, $\mathrm{O}_{2}$ uptake, and endurance. J. Appl. Physiol., 64, 1098-1106.

26. Bahr, R., Ingnes, I., Vaage, O., Sejersted, O.M., and Newsholme, E.A. (1987): Effect of duration of exercise on excess postexercise $\mathrm{O}_{2}$ consumption. J. Appl. Physiol., 62, 485-490.

27. Bahr, R., and Sejersted, O.M.: Effect of feeding and fasting on excess postexercise oxygen consumption. J. Appl. Physiol., 71, 2088-2093.

28. Geloen, A., and Trayhurn, P. (1990): Regulation of the level of uncoupling protein in brown adipose tissue by insulin requires the mediation of the sympathetic nervous system. FEBS Lett., 267, 265-267.

29. Mayes, P.A. (1993): Biosynthesis of fatty acids, in Harper's Biochemistry, 23 ed., ed. by Murray, R., Granner, D.K., Mayes, P.A., and Rodwell, V.W., Appleton \& Lange, East Norwalk, pp. 212-219.

30. Weststrate, J.W. (1993): Resting metabolic rate and diet-induced thermogenesis: A methodological reappraisal. Am. J. Clin. Nutr., 58, 592-601. 